\title{
Schwere COPD
}

\section{Nur jeder Fünfte profitiert von ICS}

_ Nur wenige Patienten mit COPD profitieren offensichtlich von inhalativen Kortikosteroiden (ICS). Eine Hilfestellung für die Therapieentscheidung gibt die Eosinophilenzahl im Blut.

GOLD empfiehlt für Patienten, die häufig exazerbieren und/oder einen $\mathrm{FEV}_{1}<50 \%$ vom Soll haben, zusätzlich zur bronchialerweiternden Erhaltungstherapie ein ICS einzusetzen [1], um das Exazerbationsrisiko zu senken.

Diese Entscheidung trägt der Beobachtung Rechnung, dass Exazerbationen nicht nur die Lebensqualität des COPDPatienten erheblich ein schränken, sondern auch eine langfristige Verschlechterung des Krankheitsverlaufs bedeuten, da sich die Lungenfunktion mit jeder Exazerbation weiter verschlechtert.
Eine auf dem Kongress der American Thoracic Society (ATS) vorgestellte Posthoc-Analyse der Studie WISDOM (Withdrawel of Inhaled Steroids During Optimised Bronchodilator Management) spricht dafür, dass selbst von den Patienten mit schwerer COPD (GOLDKlassifikation $C$ und D) nur jeder fünfte tatsächlich von der ICS-Gabe profitiert: Lediglich bei $20 \%$ dieser Patienten wurde das Exazerbationsrisiko durch die Gabe eines ICS zusätzlich zu Tiotropium (Spiriva) und einem langwirksamen $\beta_{2}{ }^{-}$ Agonisten (LABA) klinisch relevant gesenkt.

Die WISDOM-Studie hatte über 52 Wochen untersucht, welche Auswirkungen ein schrittweises Absetzen von ICS bei Patienten mit schwerer und sehr schwerer COPD und einer Exazerbationshistorie hat. Die neue Post-hocAnalyse zeigte, dass sich die wenigen Patienten, die einen Nutzen aus der zusätzlichen ICS-Anwendung ziehen, anhand der Eosinophilen-Zahl im Blut identifizieren lassen: $20 \%$ der Patienten wiesen mindestens 300 Zellen/ $\mu \mathrm{l}(\geq 4 \%)$ auf und profitierten vom ICS. Die mit $80 \%$ überwiegende Mehrheit der Patienten wies Werte $<300$ Zellen/ $\mu \mathrm{l}(\leq 4 \%)$ auf und sprach nicht statistisch signifikant auf das ICS an.

Dr. Kirsten Westphal

- 1. Global Initiative for Chronic Obstructive Lung Disease (GOLD) 2016. http://goldcopd.org/

2. Watz Het al. Blood eosinophil count and exacerbations in severechronic obstructive pulmonary disease after withdrawal of inhealed corticosteroids: a post-hoc analysis of the WISDOM trial. Lancet Resp Med. 2016;4:390-8

\section{Erkältungskrankheiten}

\section{Effektive Linderung typischer Halsbeschwerden}

_ In einer nicht-interventionellen Studie zeigte sich, dass die Kombination aus Isländisch Moos, einem Hydrogel-Komplex und Hyaluronsäure (isla med hyd$\mathrm{ro}^{+}$) rasche und anhaltende Hilfe bei den für Erkältungskrankheiten typischen Halsbeschwerden bringt. In die Studie eingeschlossen waren mehr als 280 Ärz-

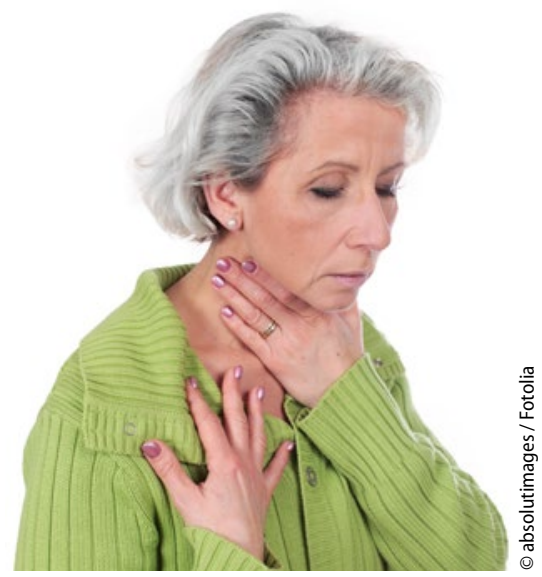

Erkältungskrankheiten heilen meist von selbst. Quälend sind die Symptome. te und 1.773 Patienten. Die entsprechenden Symptome besserten sich unter isla ${ }^{\circledR}$ med hydro ${ }^{+}$deutlich: Nach zwei bis fünf Behandlungstagen konnten die Halsschmerzen um 53,3\%, die Schluckbeschwerden um $52,4 \%$ und das Kratzen im Hals um 50,2\% reduziert werden.

\section{Schneller und lang anhaltender Effekt} Gleichzeitig wurde der allgemeine Schweregrad der Symptome deutlich reduziert. Zum Zeitpunkt der Abschlussvisite waren $89,1 \%$ der Patienten beschwerdefrei bzw. wiesen Beschwerden nur noch in leichter oder mäßiger Ausprägung auf. Dabei bewerteten sie ebenso wie die Mediziner - die Behandlung als sehr gut oder gut verträglich. Dies steht im Einklang mit bisherigen klinischen Erfahrungen zu Isländisch Moos, insbesondere einer Anwendungsbeobachtung mit 3.143 Kindern.

Ein weiteres zentrales Studienergebnis betrifft den Wirkeintritt: $84 \%$ der Patienten nahmen sofort mit dem Lut- schen oder nach nur wenigen Minuten das Eintreten eines spürbaren Effekts wahr. $83,5 \%$ gaben zudem die Empfindung eines kühlenden Effekts im Sinne einer Befreiung an. Die Wirkdauer bewerteten $73,7 \%$ der Patienten dabei mit bis zu 60 Minuten und länger.

Dies ist wahrscheinlich auf mukoadhäsive Eigenschaften zurückzuführen. $\mathrm{Zu}$ den Komponenten der Halspastillen zählen ein Spezialextrakt aus Isländisch Moos, Hyaluronsäure und ein vorgequollener Hydrogel-Komplex. Der Schleimbildner löst sich beim Lutschen balsamartig auf und legt sich wie ein Schutzfilm über die trockene Mukosa. Gleichzeitig stellt die Hyaluronsäure durch ihr hohes Wasserbindungsvermögen eine intensive Befeuchtung sicher und sorgt zusammen mit dem Quellvermögen des Hydrogel-Komplexes für ein gutes und langes Anhaften an der Schleimhaut.

Red.

- Nach Informationen von Engelhard 